\title{
SIMULATION OF SOIL-COMPACTION WITH VIBRATORY ROLLERS
}

\author{
Dieter Pietzsch, Technische Universität Berlin \\ Fachgebiet Konstruktion von Baumaschinen \\ Berlin, Deutschland
}

\begin{abstract}
Most frequently, vibratory rollers are used for compaction tasks during earth works and road construction. An increase in roller performance can be achieved by automatically adapting the machine's parameters which determine the compaction result and by automatically controlling the state of compaction continuously. The actual problem is, that because of the extensive variety in properties of the materials to be compacted and the conditions in job site, it is very difficult to state the criteria for the feedback control of the roller parameters. By means of modelling and simulating, the vibration behaviour and compaction effect as well as the interdependence between the state of roller-operation and the state of soil-compaction is investigated. The goal is to aquire knowledge about the interactions between vibratory rollers and soil during compaction in order to improve roller construction and automatic compaction measuring-systems.
\end{abstract}

Keywords: roller/soil-system, simulation, vibration behaviour, compaction effect

\section{INTRODUCTION}

Vibratory rollers are primarily used for compaction tasks in earthwork and road building. The benefits of dynamic compaction led to vibratory rollers taking a share of more than $90 \%$ of the total roller market. The compaction effect and performance of these machines depends on their features and technical data as well as on the properties of the building material and the conditions during building-in [1].

An increase in roller performance can be achieved on the one hand by automation of the compaction process (that means adapting the machine's parameters automatically to the given conditions on the construction site), and on the other hand by monitoring the compaction process with a continuously-working measuring-system in situ.

Because the properties of the soil usually can not significantly be influenced, the progress in compaction and the compaction result have to be controlled by adjusting the machine's parameters, i.e. excitation frequency and force, roller speed and number of roller passes.

The automatic feedback control of the roller parameters in operation, influenced through the hydrostatic drives for excitation force and roller speed, can be done comparatively simply by the means of micro-electronics. The actual problem is, that the criteria for the feedback control of the roller parameters are not known, and changes during compaction, i.e. disturbances which depend on the building material, cannot be detected securely. 
Before a non-destructive mechanical measuring-system for compaction can be developed, the correlation between a measurable quantity at the drum and the state of soil-compaction must be verified. Although several roller manufacturers have been offering dynamic test equipment for assessing an overall state of compaction during operation for several years, no universally valid connection between the measured drum acceleration and the dynamic stiffness or rather the state of soil-compaction can be stated until now.

Solving the named problems requires a mathematical description of the interdependence between the state of roller operation and the state of compaction of the building material. For this purpose a calculation model for simulating all states of operation of the roller/soil-system is necessary. This model is the foundation for all calculations and simulations for investigating various influences on vibration behaviour of the roller and the compaction progress of the building material.

\section{MATHEMATICAL MODEL}

The dynamic behaviour of vibratory rollers is determined by the combination of the machine and the soil to be compacted.

Models suitable for calculating the dynamic behaviour of the roller/soil-system must enable the investigation of all important phenomena. The mathematical description of the interaction between roller and soil during compaction requires an analytical model comprising:

- an analytical model for the roller and

- a mathematical model which describes the qualities of the soil, relevant to compaction.

These analytical models can either be built as a set of discrete mechanical elements such as masses, springs and dampers or be described as continua. The decision on the model concept depends on the specific demands relevant to the dynamic behaviour of the two parts of the roller/soil-model, the acceptable volume of calculations and the precision of the simulated system characteristics. In order to calculate the vibration and compaction behaviour of the model, the parameter values have to be determined. The design of the calculation model therefore additionally depends on the existing possibilities and methods for parameter determination.

Because of the complex effects inside the soil it is not possible to describe the internal processes during compaction mathematically. Therefore, all model based calculations can only approximate the actual vibration and compaction behaviour.

The design of the model, together with the explanation of the elements and the definition of motion coordinates, is shown in Fig. 1.

The model of the roller consists of the drum mass $m_{d}$, the frame mass $m_{f}$ and the frame suspension with the spring $k_{f}$ and the damper $d_{f}$. The drum is set into vibration by the excitation force $F_{e}$, rotating with the angular velocity $\Omega$. With this model tow type rollers as well as self-propelled rollers can be simulated. The influence of the 
inertial moment of the frame mass of self-propelled rollers on the vibrating behaviour must be considered by an equivalent frame mass $m_{f}{ }^{*}$.

The primary demand on the soil model is that the plastic and elasto-plastic deformations can be described with it. An increase in plastic deformation (compaction) of the soil can only be achieved by increasing the effective force. This force must depend on the displacement of the soil and, in order to transform energy, counteract the effect of the loading velocity. This law for the force can be met with a spring model consisting of three springs. Two springs - the springs $\mathrm{k}_{\mathrm{pl}}$ and $\mathrm{k}_{\mathrm{pl}}$ ' - click into place at the points of maximum deformation [2]. Because of that any further plastic deformation occurs only when the spring $\mathrm{k}_{\mathrm{el}}$ is deformed so far that the maximum displacement of the previous loading is reached again. As a result, a continually increasing portion of the kinetic energy of the drum is received only by the spring $\mathrm{k}_{\mathrm{el}}$ and returned to the drum during relieving.

The springs which click into place can be described as having lateral guidances allowing motion in only one direction. If these springs are compressed, the guidances cause the springs to arrest in the positions of maximum deformation. The energy deforming the springs is thus stored therein.

In addition to this structure of springs, the soil model includes a mass $m_{s}$ representing the mass of the soil caused to vibrate by the drum and a system with the mass $m_{a}$, spring $k_{a}$ and damper $d_{a}$, necessary to control the motion of the soil mass during bounce operation of the drum.

\subsection{MODES OF ROLLER OPERATION}

During compaction the mode of roller motion is distinguished as contact and bounce operation, because the drum leaves the ground when the vibrations exceed a certain intensity. The mode of drum motion at a certain time depends on the compaction force, the force transmitted in the contact area between drum and soil. Investigations by Jurecka [3] and Dimpfl [4] show that the ratio of the maximum excitation force to the weight of the vibrating mass is not the only criterion in defining contact and bounce operation. Drum lift-off also depends on the suspension, damping and the vibrating mass of the compacted soil. The division of drum motion into contact and bounce operation is illustrated in Fig. 2. This illustration shows, that during contact operation of the drum two different states of soil deformation are possible:

- contact operation 1

During an elasto-plastic deformation all springs of the soil model are under load. The springs $\mathrm{k}_{\mathrm{pl}}$ and $\mathrm{kpl}_{\mathrm{pl}}$ are irreversibly and the spring $\mathrm{k}_{\mathrm{el}}$ is reversibly

- contact operation 2

During the elastic soil deformation only the spring $k_{e l}$ is deformed, while springs $k_{\mathrm{pl}}$ and $\mathrm{k}_{\mathrm{pl}}$, have already reached their maximum compression. 
During bounce operation the drum is lifted off the soil. The masses of soil and drum are not in contact and the soil is not compacted. The next contact operation begins with an impact between drum and soil.

The described modes of drum motion and the types of soil deformation are present during compaction, each with different duration and frequency [5]. The calculation of non-linear drum motion and soil deformation for the duration of compaction means it is necessary to skip mathematically between the differential equation systems describing the actual modes of operation. At the same time transfer and boundary conditions between the differential equation systems must be considered. This problem is numerically solved by a calculation program.

The differential equations for the different modes of roller operation are formulated in matrix-form below. The excitation force is calculated from the mass and size of the eccentric and it's angular velocity $\Omega$ :

$$
F_{e}=m_{u} \cdot r_{u} \cdot \Omega^{2} \cdot \sin (\Omega t)
$$

The eccentric moment $m_{u} r_{u}$ directly determines the nominal amplitude of the drum displacement. The force transmitted by the rubber elements between drum and frame of the roller is independent of the state of roller operation:

$$
F_{f}=k_{f} \cdot\left(z_{f}-z_{d}\right)+d_{f} \cdot\left(\dot{z}_{f}-\dot{z}_{d}\right)
$$

Equation system for contact operation 1

$$
\begin{aligned}
& {\left[\begin{array}{ccc}
m_{f} & 0 & 0 \\
0 & \left(m_{d}+m_{s}\right) & 0 \\
0 & 0 & m_{a}
\end{array}\right] \cdot\left[\begin{array}{l}
\ddot{z}_{f} \\
\ddot{z}_{d} \\
\ddot{z}_{a}
\end{array}\right]+\left[\begin{array}{ccc}
d_{f} & -d_{f} & 0 \\
-d_{f}\left(d_{f}+d_{a}\right) & -d_{a} \\
0 & -d_{a} & d_{a}
\end{array}\right] \cdot\left[\begin{array}{l}
z_{f} \\
\dot{z}_{d} \\
\dot{z}_{a}
\end{array}\right]+} \\
& {\left[\begin{array}{ccc}
k_{f} & -k_{f} & 0 \\
-k_{f} & k^{*} & -k_{a} \\
0 & -k_{a} & k_{a}
\end{array}\right] \cdot\left[\begin{array}{c}
z_{f} \\
z_{d} \\
z_{a}
\end{array}\right]=\left[\begin{array}{c}
m_{f} \cdot g \\
\left(m_{d}+m_{s}\right) \cdot g \\
m_{a} \cdot g
\end{array}\right]+\left[\begin{array}{l}
0 \\
F_{e} \\
0
\end{array}\right]}
\end{aligned}
$$

$$
\text { and } k^{*}=\left(\left(k_{e l}+k_{p l}{ }^{\prime}\right) \cdot k_{p l} /\left(k_{e l}+k_{p l}{ }^{\prime}+k_{p l}\right)\right)+k_{a}+k_{f}
$$


Equation system for contact operation 2

$\left[\begin{array}{ccc}m_{f} & 0 & 0 \\ 0 & \left(m_{d}+m_{s}\right) & 0 \\ 0 & 0 & m_{a}\end{array}\right] \cdot\left[\begin{array}{c}\ddot{z}_{f} \\ \ddot{z}_{d} \\ \ddot{z}_{a}\end{array}\right]+\left[\begin{array}{ccc}d_{f} & -d_{f} & 0 \\ -d_{f} & \left(d_{f}+d_{a}\right) & -d_{a} \\ 0 & -d_{a} & d_{a}\end{array}\right] \cdot\left[\begin{array}{c}\dot{z}_{f} \\ \dot{z}_{d} \\ \dot{z}_{a}\end{array}\right]+$

$\left[\begin{array}{ccc}k_{f} & -k_{f} & 0 \\ -k_{f} & k^{*} & -k_{a} \\ 0 & -k_{a} & k_{a}\end{array}\right] \cdot\left[\begin{array}{l}z_{f} \\ z_{d} \\ z_{a}\end{array}\right]=\left[\begin{array}{l}m_{f} \cdot g \\ \left(m_{d}+m_{s}\right) \cdot g+\dot{F}_{e}+k_{e l} \cdot z_{v} \\ m_{a} \cdot g\end{array}\right]$

and $k^{*}=k_{e l}+k_{f}+k_{a}$

Equation systems for bounce operation

Equation system for the roller:

$$
\begin{aligned}
& {\left[\begin{array}{cc}
m_{f} & 0 \\
0 & m_{d}
\end{array}\right] \cdot\left[\begin{array}{l}
\ddot{z}_{f} \\
\ddot{z}_{d}
\end{array}\right]+\left[\begin{array}{cc}
d_{f} & -d_{f} \\
-d_{f} & d_{f}
\end{array}\right] \cdot\left[\begin{array}{l}
\dot{z}_{f} \\
\dot{z}_{d}
\end{array}\right]+\left[\begin{array}{cc}
k_{f} & -k_{f} \\
-k_{f} & k_{f}
\end{array}\right] \cdot\left[\begin{array}{l}
z_{f} \\
z_{d}
\end{array}\right]} \\
& =\left[\begin{array}{c}
m_{f} \cdot g \\
m_{d} \cdot g
\end{array}\right]+\left[\begin{array}{c}
m_{u} \cdot r_{u} \cdot \Omega^{2} \cdot \sin (\Omega t)
\end{array}\right]
\end{aligned}
$$


Equation system for the soil deformation:

$$
\begin{aligned}
& {\left[\begin{array}{ll}
m_{s} & 0 \\
0 & m_{a}
\end{array}\right] \cdot\left[\begin{array}{l}
\ddot{z}_{s} \\
\ddot{z}_{a}
\end{array}\right]+\left[\begin{array}{ll}
d_{a} & -d_{a} \\
-d_{a} & d_{a}
\end{array}\right] \cdot\left[\begin{array}{l}
\dot{z}_{s} \\
\dot{z}_{a}
\end{array}\right]+\left[\begin{array}{ll}
k^{*} & -k_{a} \\
-k_{a} & k_{a}
\end{array}\right] \cdot\left[\begin{array}{l}
z_{s} \\
z_{a}
\end{array}\right]} \\
& =\left[\begin{array}{l}
m_{s} \cdot g+k_{e l} \cdot z_{v} \\
m_{a} \cdot g
\end{array}\right]
\end{aligned}
$$

$$
\text { and } k^{\star}=k_{e l}+k_{a}
$$

\subsection{MODEL PARAMETERS}

For running the simulation program, it is necessary to determine the values of the masses, spring stiffnesses and damping factors of the model.

\section{Roller-Parameters}

For the roller the technical data of a specific machine can be used as model parameters or, if a new machine is designed, the parameters can be chosen freely. The vibrating mass and the frame mass of tow type rollers can easily be determined. For self-propelled vibratory rollers with two pneumatic drive wheels and for tandem vibratory rollers it is necesseary to determine the equivalent frame mass $m_{f}$ :

$$
m_{f}^{*}=\frac{\Theta_{y R}+m_{f} \cdot\left(l_{h}^{2}+h_{s}^{2}\right)}{l_{r}^{2}}
$$

\footnotetext{
$m_{f}$ Frame mass

I Distance centre of mass - rear axle

$I_{r}$ Distance middle of drum - rear axle

$h_{s}$ Height of mass centre - soil-surface

$\theta_{\mathrm{yR}}$ Moment of inertia of roller
} 
The equivalent frame mass can be derived from the inertial moment $\theta_{\mathrm{yR}}$ of the frame mass. Values for the inertial moment are not included in the technical specifications given by the manufacturers. It must be determined by measuring the period of the free undamped vibration of the whole machine.

The frames of vibratory rollers are usually isolated from the drum by rubber elements. These elements have to transmit the dynamic force generated by the eccentric moment and, depending on the type of roller, additional steering forces and the driving moment. In order to calculate the vibrating behaviour of rollers, the static and dynamic shear stiffness and the damping of the rubber elements are required.

\section{Soil-Parameters}

For the soil model the vibrating soil mass, the stiffness of springs and the parameters of the additional system must be determined. In contrast to the determination of the roller parameters, it is much more difficult to determine the parameters of the soil model because there is no mathematical connection between the actual properties of the soil and the model parameters. Additionally, the dependencies of the model parameters on influencing quantities are not known [6]. Because the total soil stiffness and the vibrating soil mass cannot be derived directly from soil properties, the model parameters must instead be calculated from measurable characteristics of the roller/ soil-system, i.e. static soil deformation, natural frequency or time responses of the drum and frame acceleration.

\section{NUMERICAL SIMULATION}

In addition to the specific computer environment (operating system, editor, utilities), the numerical simulation of the roller/soil-system requires programs for various tasks:

- Simulation program for the roller/soil-system (FORTRAN)

- Additional programs for initialisation, parameter preparation and batch control of program execution

- Programs for evaluation and reduction of the results in the time domain and for frequency analysis

- Programs for the graphic presentation of the results

Of these programs, only the simulation program will be described in detail. The differential equations of the different modes of roller operation are the basic equations for the simulation of the model. These equations are solved by a simulation program which uses FORTRAN-subroutines from a mathematical library (IMSL) [7]. The flowchart shown in Fig. 3 represents the structure of this program. The transitions from contact operation to bounce operation and from elasto-plastic to elastic soil deformation are controlled by internal program decisions. 
When the program is started, the following files, which are needed while pro-. gram is running, will be opened:

- INIVAL includes the parameters of roller and soil and the time boudaries for the numerical integration routines

- PARSET includes a list of system characteristics when program is finished

- DATA 1 and

- DATA 2 include the results of the model characteristics in the time domain

The subroutine PARARS reads the parameters of the roller and soil from the file INIVAL. These are pre-processed masses, spring stiffnesses and damping factors as well as the moment of unbalance and the rotational speed of the rotating eccentric. The subroutine INICON provides the initial conditions for the displacements and velocities to the integration routines. With the subroutine INTDAT the data for the integration loop, start time (BEG), end time (END) and the time step (DELT) for the integration are determined using the file INIVAL. The most important part of the program is the integration loop. In this loop the displacements, velocities and accelerations of the model masses are calculated for each time step DELT. Inside this loop the different states of the model motions are controlled and, according to the specific mode of operation, the vibrating behaviour of the roller and the state of soil compaction is calculated within the following subroutines:

- contact operation 1 during the elasto-plastic soil deformation (compaction)

- contact operation 2 during the elastic soil deformation

- bounce operation

While changing from bounce to contact operation, there is an impact between drum and soil. The change in velocity as a result of this impact is calculated within the subroutine IMPULS. After every program-pass for the time step DELT the subroutine OUTPUT writes the results to the files DATA 1 and DATA 2.

\section{RESULTS}

Simulation is carried out for the system of self-propelled vibratory roller/soil. The determination of the parameters for the roller and soil model is based on the technical data of the roller and on preset data concerning the frame suspension and the natural frequency of the system as combined in Table 1. The calculated parameters for the roller and soil model are shown in Table 2 and 3.

The properties of the results are included in the amplitude and frequency response of the time series as well as in the curve's shape. As an example the calculated time series at the operation frequency $f_{e}=30 \mathrm{~Hz}$ are shown for the displacements of the drum, frame and soil in Fig. 4 and for the accelerations of frame and drum in 
The displacement-time diagram in Fig. 4 shows, that there are time domains where drum and soil-mass are vibrating conformally (contact operation) and domains where drum and soil-mass have different displacements (bounce operation). Corresponding to the frequency ratio $\eta=3.0$ the frame mass is vibrating mainly with the natural frequency $f_{\text {of }}=10 \mathrm{~Hz}$. Further calculations indicate that the displacement amplitudes of the frame become greater with increasing frequency ratio $\eta$ and that the time domain of bounce operation increases with the natural system frequency $f_{0 e p}$ getting nearer to the excitation frequency $f_{e}$ of the roller.

The acceleration-time diagram in Fig. 5 shows that the curve of the drum acceleration is not entirely sinusoidal, but becomes distorted at certain times. These distortions are caused by the changes in the mode of drum operation, i.e. when the drum hits the soil at the end of the bounce operation. The diagram in Fig. 5 and further simulations show that the amplitudes of the calculated accelerations of drum and frame and the shape of the curves are fully comparable to acceleration signals measured on soils with different states of compaction.

The frequency ratio $\eta$ between the excitation frequency $f_{e}$ and the natural frequency of the frame mass $f_{\text {of }}$ can be varied by installation of rubber elements with a different Shore Hardness. Fig. 6 shows the RMS-values of the frame acceleration plotted against the excitation frequency and different frequency ratios. By means of this time data reduction the values of the results can be compared at the operation frequency $f_{e}=30 \mathrm{~Hz}$, and the effects of various excitation frequencies on the results can be determined.

At the operation frequency $f_{e}=30 \mathrm{~Hz}$ of the roller the acceleration of the frame decreases with the frequency ratio $\eta$ becomming greater. In the frequency range corresponding with the natural frequencies of the frame mass, no resonance vibration can be realized. The reasons are, that on one hand the vibration excitation in this frequency range is very low because of the quadratic increase in centrifugal force with increasing rotational speed of the eccentric, and on the other hand the frame vibration is damped by the reaction of the mass of inertia of the roller. Resonant frame vibration can occur in the range between the natural frequency of the frame mass and the operation frequency. The smaller the difference between these frequencies is, the greater the frequency range with resonant frame vibration is. Each hatched area in Fig. 6 is restricted by curves of frame accelerations which are calculated with a constant spring stiffness of the rubber elements (dotted line) and with frequency dependent spring stiffness of the rubber elements, i.e. solid line for $\eta=5.0$. The values of the frame acceleration for constant and frequency dependent spring stiffness are different
mainly in the range of resonant frame vibration.

The RMS-values of the drum acceleration plotted against the excitation frequency are shown in Fig. 7 , with the excitation force $F_{e}$ as parameter. At the operation frequency $f_{e}=30 \mathrm{~Hz}$ of the roller, the drum acceleration amplitudes increase with the excitation force becomming greater with respect to the static drum weight. The solid line in Fig. 7 represents the calculated drum acceleration of the actual roller with an installed excitation force $F_{e}=105 \mathrm{kN}$. Around the operation frequency the course of the drum acceleration is nearly constant, therefore the values of the drum acceleration are independent on variations of the excitation frequency. With the amplitude of the 


\section{SUMMARY}

The combination of a vibratory roller and soil represents a closed-loop system. For the reactions of this system, differences between reasons and effects cannot be stated separately. By means of modelling and simulation, the vibrating behaviour as well as the compaction effect of vibratory rollers is investigated. For the representation of the interdependencies between roller and soil during compaction a calculation model is developed, consisting of two models. One is an analytical model for the roller, the other is a model which describes the qualities of the soil, relevant to compaction. When defining the mathematical model, the non-linearities of roller motion and ground deformation are considered through a distinction between bounce and contact operation as well as between elastic and non-elastic ground deformation.

Simulation results verify that the calculation model for the roller/soil-system is suitable for mathematically determining the vibration behaviour and the compaction effect of the roller. The simulation accordingly permits the computer-based trial of closed loops to automatically optimize the constructional and operational roller parameters. Furthermore, the quality of goal-directed design of vibratory rollers can be enhanced before prototypes are built. Expensive iteration loops between design and experiment can, therefore, be avoided.

\section{REFERENCES}

[1] Forssblad, L.: Vibratory Soil and Rock Fill Compaction Dynapac Maskin AB, Solna Sweden, 1981

[2] Bathelt, U.: Das Arbeitsverhalten des Rüttelverdichters auf plastischelastischem Untergrund Bautechnik Archiv, Heft 12, Berlin: Verlag Wilhelm Ernst \& Sohn, 1956

[3] Jurecka, W.: Das Bewegungsverhalten und die Wirkungsweise von Rüttelverdichtern

Baumaschine und Bautechnik 18 (1971) 11, S. 461-466

[4] Dimpfl, G.: Untersuchungen über den Energieaustausch bei der Bodenverdichtung mit Sprungrüttlern Dissertation, RWTH Aachen, 1965

[5] Kröber, W.: Untersuchung der dynamischen Vorgänge bei der Vibrationsverdichtung von Böden Dissertation, TU München, 1988

[6] Yoo, T.-S., Selig, E.T.: Dynamics of Vibratory-Roller-Compaction Journal of the Geotechnical Engineering Division, No. 105 (1979) 10, S. $1211-1231$ 


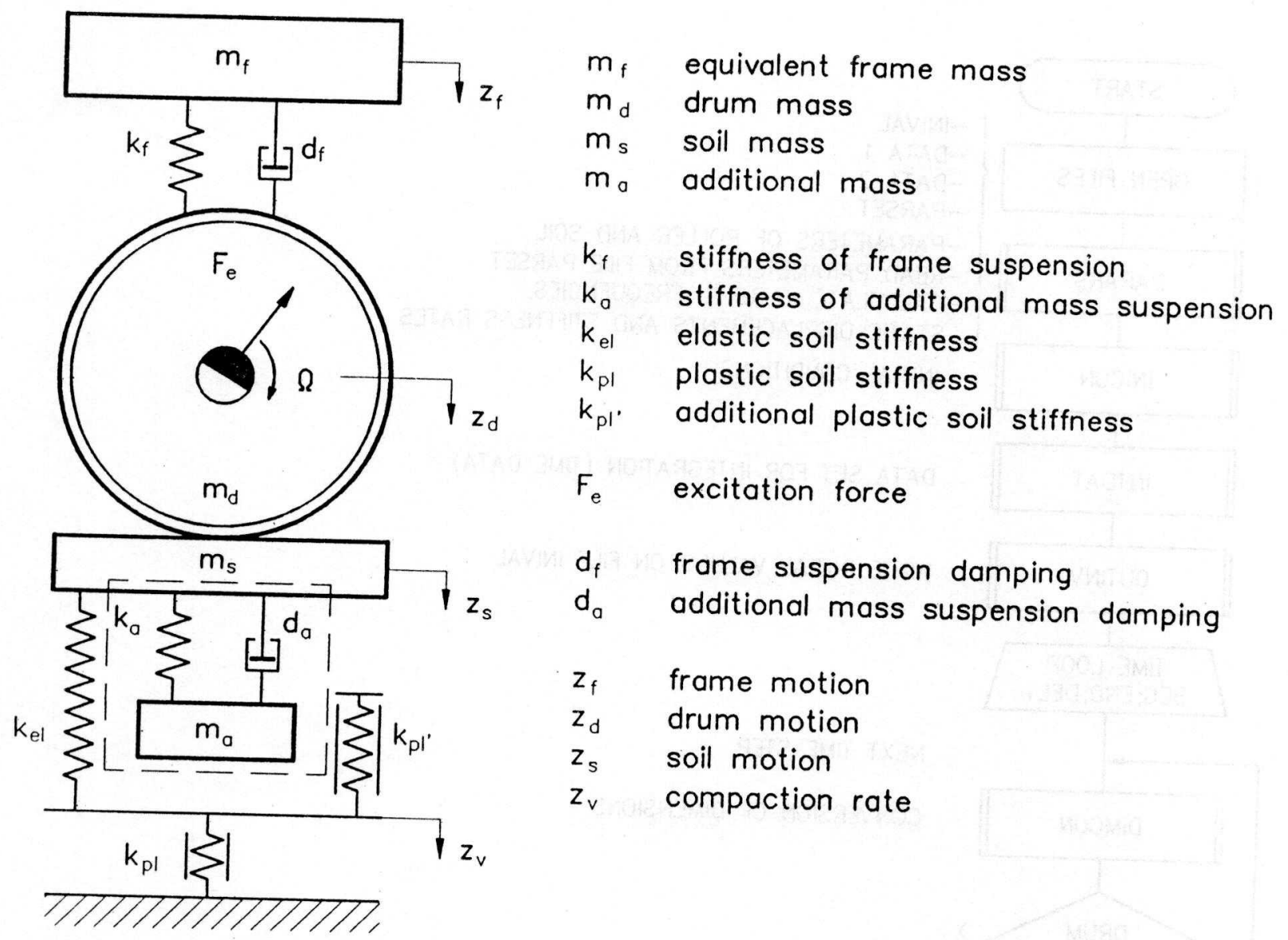

Fig. 1: Analytical model for the roller/soil-system

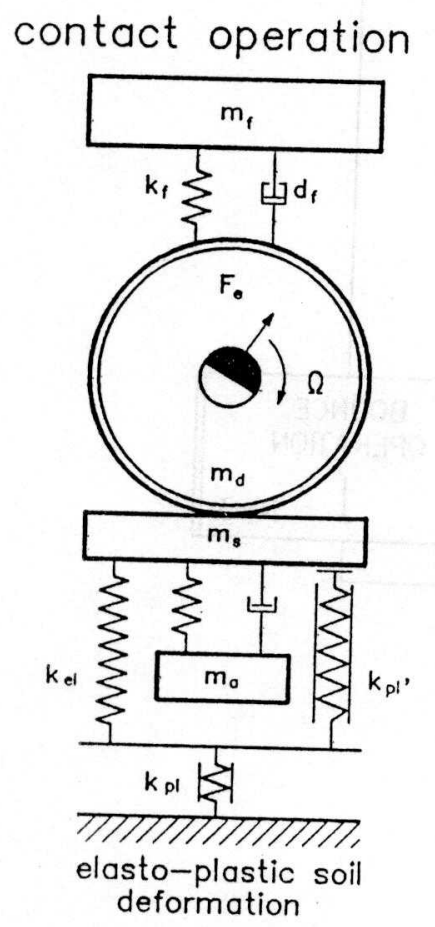

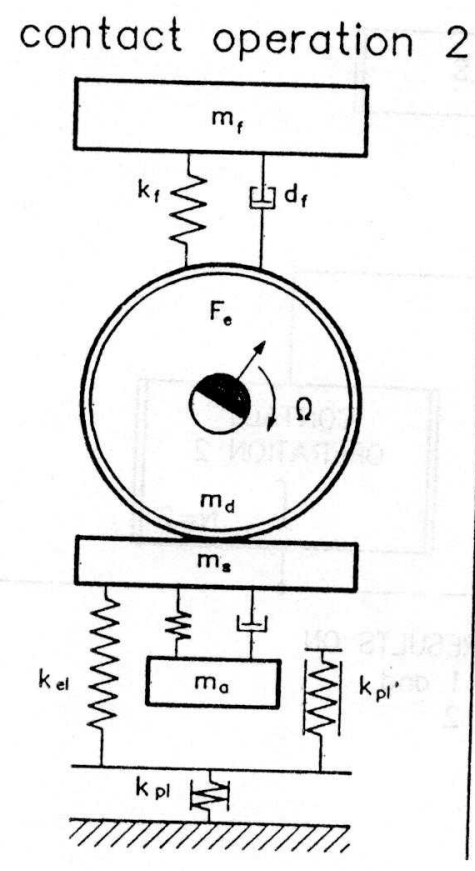

elastic soil-deformation bounce operation

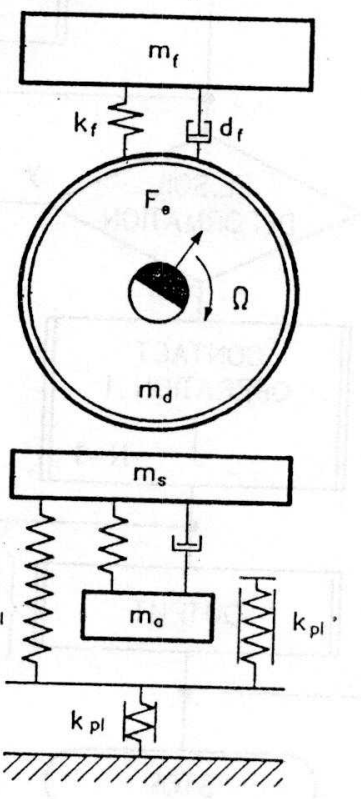

elastic soil-deformation

Fig. 2: Illustration of contact and bounce operation of drum 


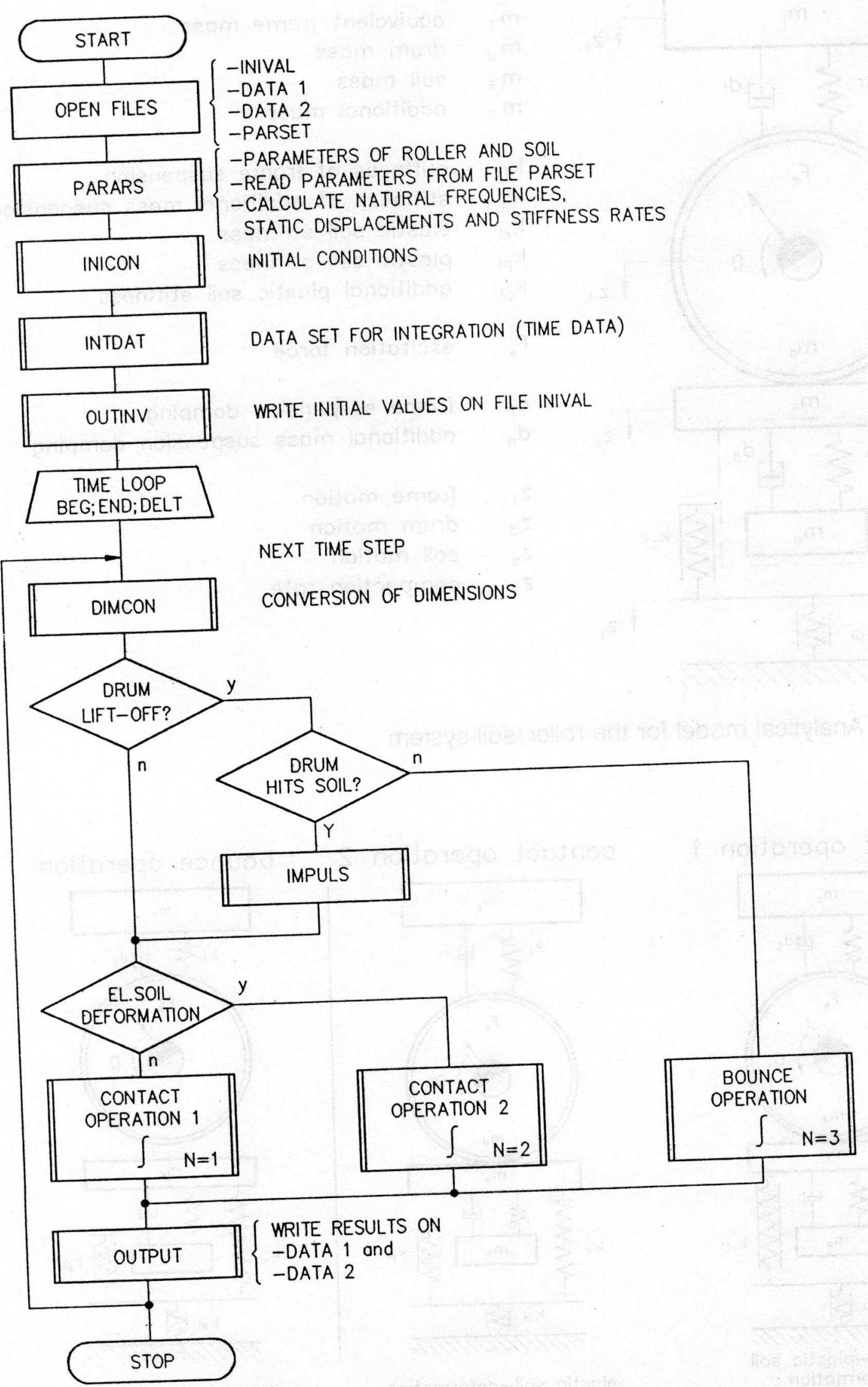

Fig. 3: Program-flowchart for simulation of the dynamic roller-behaviour 


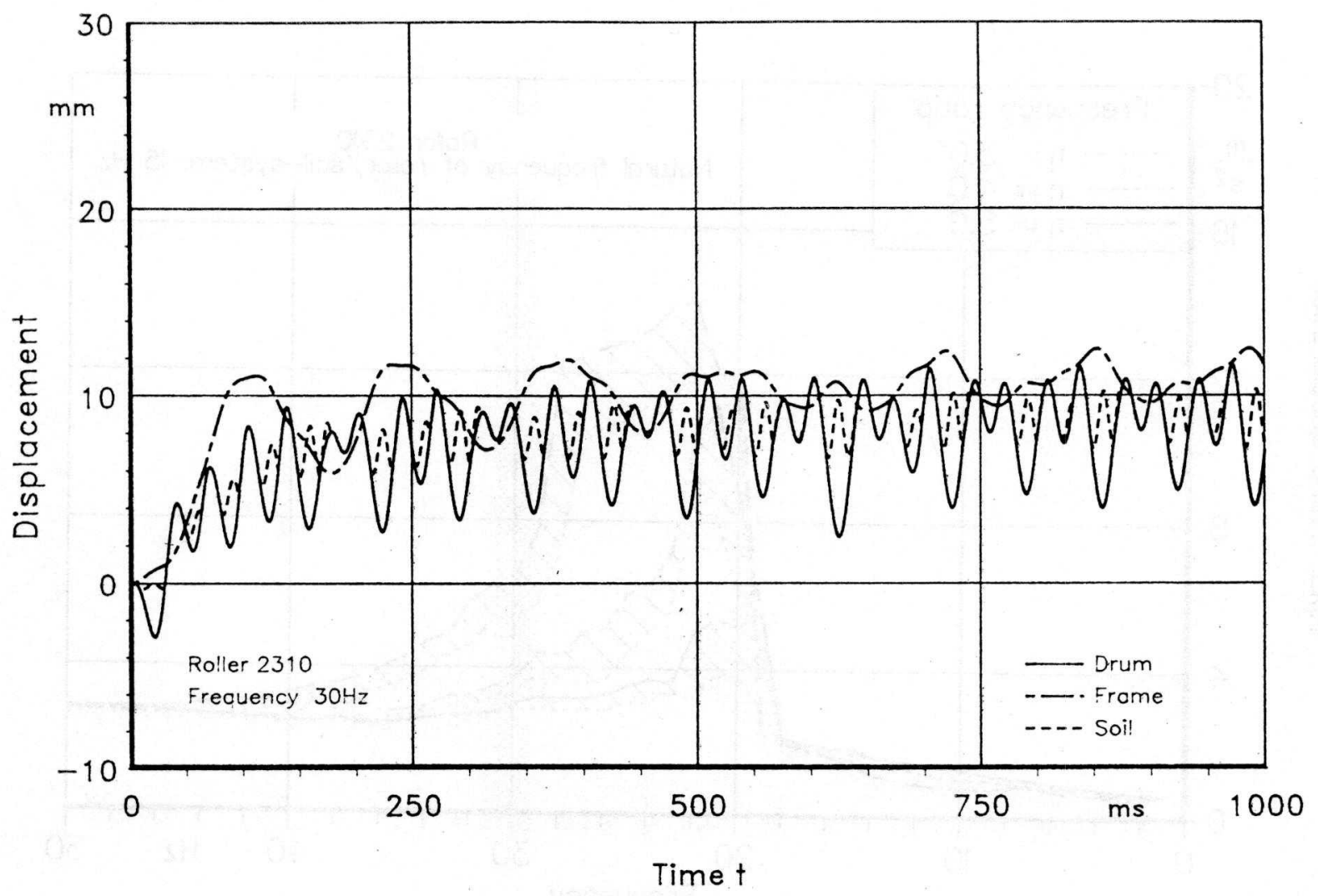

Fig. 4: Displacements of drum, frame and soil $\left(\mathrm{f}_{\mathrm{e}}=30 \mathrm{~Hz}, \eta=3.0, \mathrm{f}_{0 \mathrm{ep}}=15 \mathrm{~Hz}\right)$

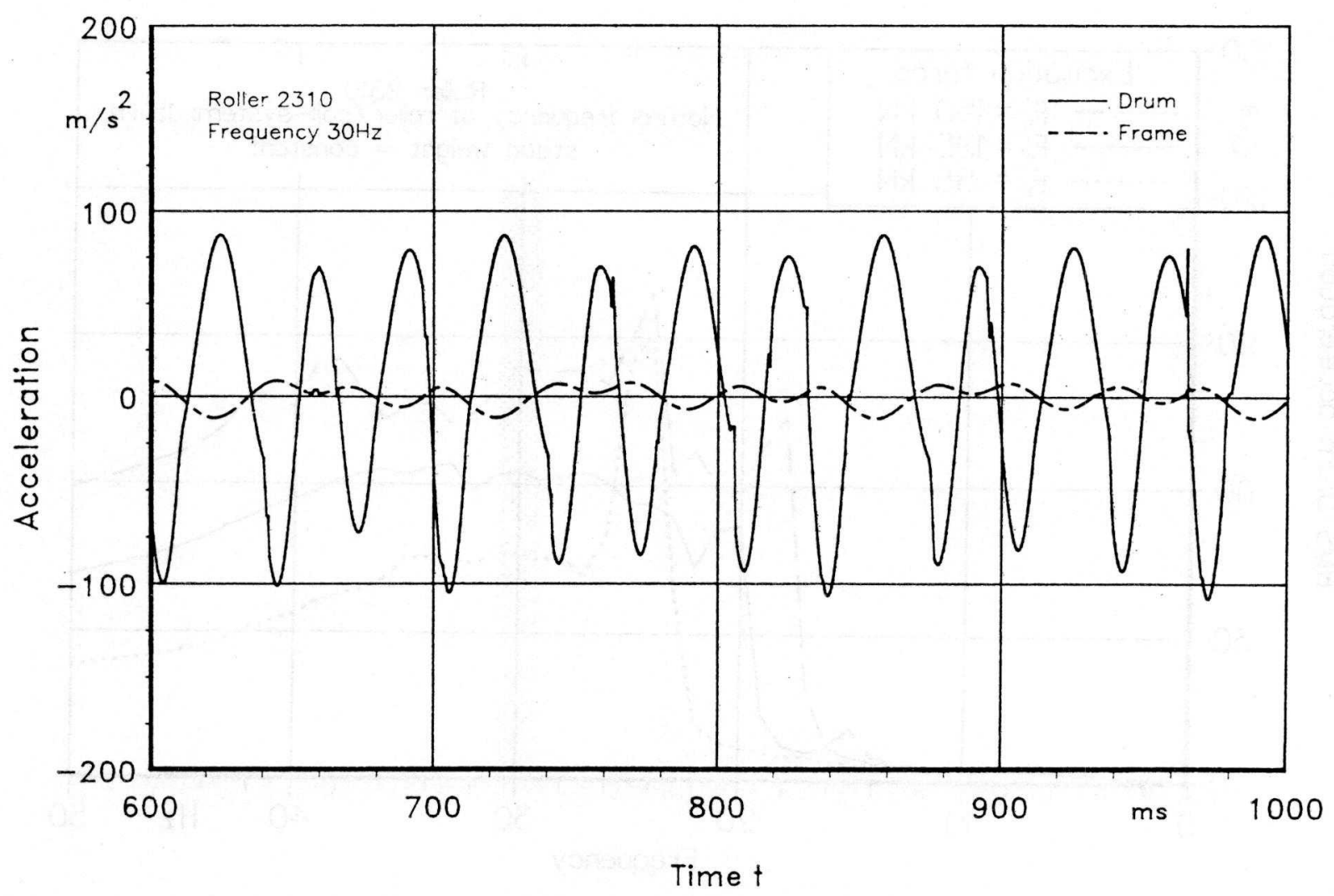

Fig. 5: Acceleration of drum and frame $\left(f_{0}=30 \mathrm{~Hz}, \eta=3.0, f_{0 n n}=15 \mathrm{~Hz}\right)$ 


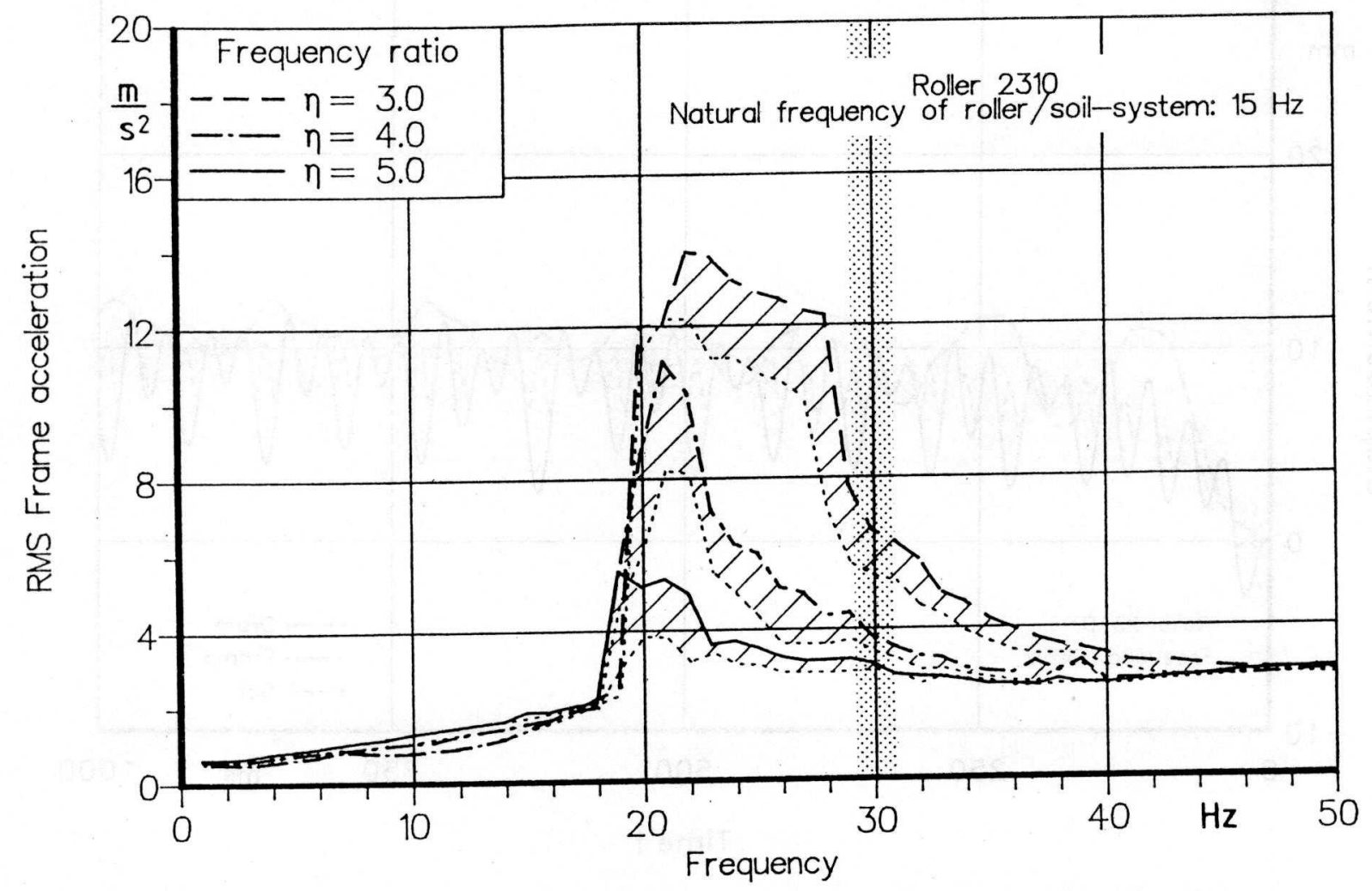

Fig. 6: RMS frame acceleration plotted against the excitation frequency with the frequency ratio as parameter

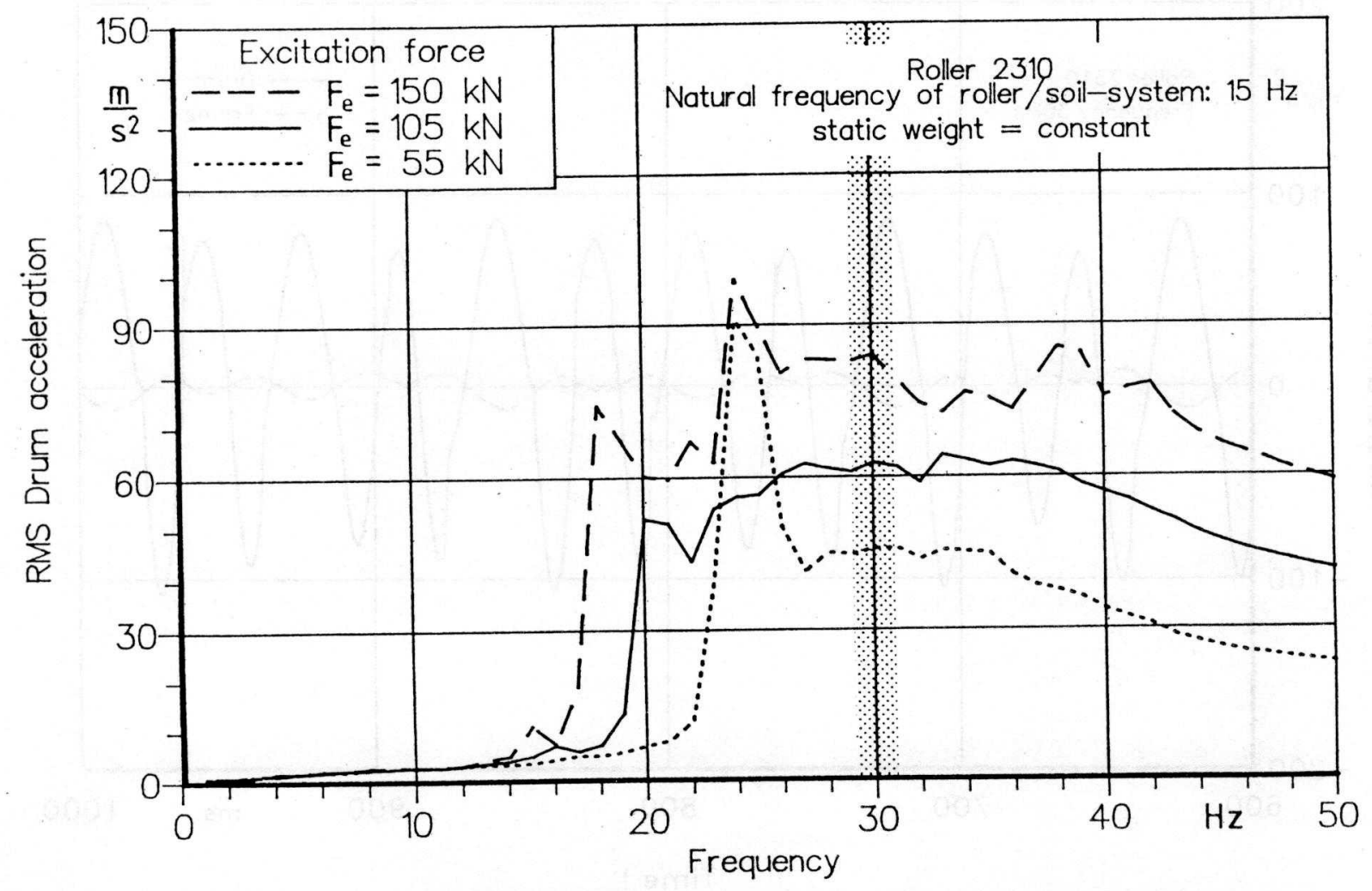

Fig. 7: RMS drum acceleration plotted against the excitation frequency with 
Table 1: Technical Data of the roller and preset data

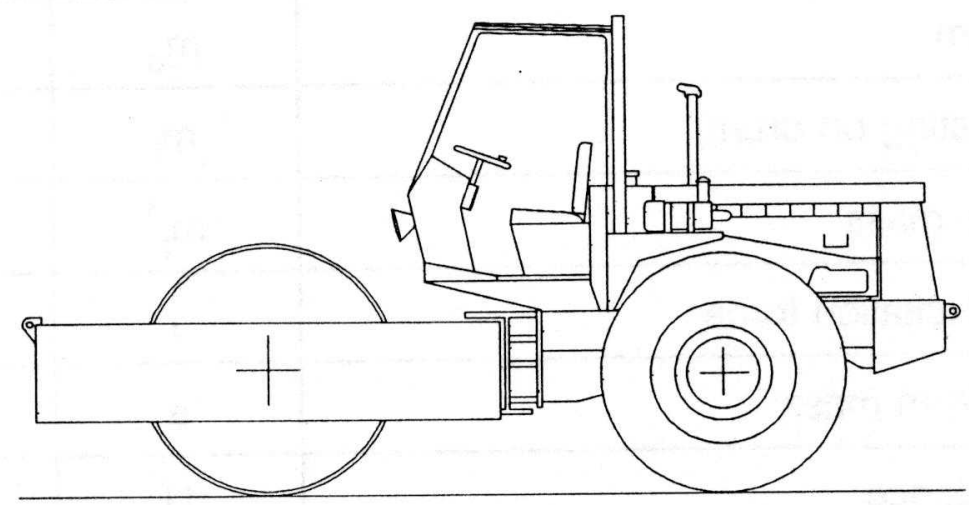

Self-propelled vibratory roller 2310 (HAMM)

Technical data provided by the manufacturer

\begin{tabular}{|c|c|c|}
\hline Operating weight & $m_{\text {tot }}$ & $6500 \mathrm{~kg}$ \\
\hline Static weight (drum) & $\mathrm{m}_{\text {stat }}$ & 3500 kg \\
\hline Excitation frequency & $f_{e}$ & $30 \mathrm{~Hz}$ \\
\hline Excitation force $\left(\mathrm{f}_{\mathrm{e}}=30 \mathrm{~Hz}\right)$ & $F_{e}$ & $105 \mathrm{kN}$ \\
\hline Nominal amplitude of drum displacement & $A_{0}$ & $1.4 \mathrm{~mm}$ \\
\hline \multicolumn{3}{|c|}{ Data from experiments } \\
\hline Distance centre of mass - rear axle & $I_{h}$ & $1.33 \mathrm{~m}$ \\
\hline Distance middle of drum - rear axle & $I_{r}$ & $2.44 \mathrm{~m}$ \\
\hline Height of mass centre - soil-surface & $h_{s}$ & $0.79 \mathrm{~m}$ \\
\hline Moment of inertia of roller & $\theta_{\mathrm{yR}}$ & $16.6 \cdot 10^{3} \mathrm{kgm}^{2}$ \\
\hline \multicolumn{3}{|c|}{ Preset data } \\
\hline Frequency ratio & $\eta$ & 3.0 \\
\hline Damping & $\mathrm{D}$ & 0.06 \\
\hline Natural frequency of system roller/soil & $f_{0 e p}$ & $15 \mathrm{~Hz}$ \\
\hline Natural frequency of additional system & $f_{a}$ & $23 \mathrm{~Hz}$ \\
\hline
\end{tabular}


Table 2: Parameters of the roller model

\begin{tabular}{|l|c|c|}
\hline \multicolumn{3}{|c|}{ Calculated data for the roller model } \\
\hline Mass of the drum & $m_{d}$ & $2110 \mathrm{~kg}$ \\
\hline Frame mass (resting on drum) & $m_{f}$ & $1390 \mathrm{~kg}$ \\
\hline Equivalent frame mass & $m_{f}^{*}$ & $2800 \mathrm{~kg}$ \\
\hline Static weight : Excitation force & $\gamma$ & 0.33 \\
\hline Frame mass : Drum mass & $\alpha$ & 0.66 \\
\hline Moment of unbalance & $U$ & $2.96 \mathrm{kgm}$ \\
\hline Natural frequency of frame mass & $\mathrm{f}_{\text {of }}$ & $15 \mathrm{~Hz}$ \\
\hline Spring stiffness of frame suspension & $k_{\mathrm{f} \text { stat }}$ & $5.48 .10^{6} \mathrm{~N} / \mathrm{m}$ \\
\hline Damping factor of frame suspension & $d_{f}$ & $10480 \mathrm{Ns} / \mathrm{m}$ \\
\hline
\end{tabular}

Table 3: Parameters of soil model

\begin{tabular}{|l|c|c|}
\hline \multicolumn{3}{|c|}{ Parameters of soil model } \\
\hline Soil mass & $\mathrm{m}_{\mathrm{s}}$ & $700 \mathrm{~kg}$ \\
\hline Additional mass & $\mathrm{m}_{\mathrm{a}}$ & $70 \mathrm{~kg}$ \\
\hline Stiffness of elastic spring & $\mathrm{k}_{\mathrm{el}}$ & $87 \cdot 10^{6} \mathrm{~N} / \mathrm{m}$ \\
\hline Stiffness of plastic spring & $\mathrm{k}_{\mathrm{pl}}$ & $49.3 \cdot 10^{6} \mathrm{~N} / \mathrm{m}$ \\
\hline Stiffness of plastic spring & $\mathrm{k}_{\mathrm{pl}}$ & $75.8 \cdot 10^{6} \mathrm{~N} / \mathrm{m}$ \\
\hline Spring stiffness of additional mass suspension & $\mathrm{k}_{\mathrm{a}}$ & $1.46 \cdot 10^{6} \mathrm{~N} / \mathrm{m}$ \\
\hline Damping factor of additional mass suspension & $\mathrm{d}_{\mathrm{a}}$ & $3440 \mathrm{Ns} / \mathrm{m}$ \\
\hline
\end{tabular}

